\title{
Parental demographics and preschool childrens theory of mind
}

\begin{abstract}
This research investigates the difference between theory of mind (ToM) and parental demographics of preschool children. One hundred and sixty six 3.6- to- 5.6 year old children were tested on three standard false belief tasks and a verbal ability test. A significant difference was found between mother's occupational status and ToM. However, hierarchical regression analysis showed that mother's occupational status was a significant predictor of children's ToM development after controlling for age and verbal ability.
\end{abstract}

Keyword: Theory of mind; False belief; Family background; Preschooler 